\title{
Approximate Periodic Solutions for Oscillatory Phenomena Modelled by Nonlinear Differential Equations
}

\author{
Constantin Bota, Bogdan Căruntu, and Olivia Bundău \\ "Politehnica" University of Timişoara, Department of Mathematics, Piata Victoriei 2, 300006 Timişoara, Romania \\ Correspondence should be addressed to Bogdan Căruntu; bogdan.caruntu@upt.ro
}

Received 14 January 2014; Accepted 29 March 2014; Published 23 April 2014

Academic Editor: Baocang Ding

Copyright ( 2014 Constantin Bota et al. This is an open access article distributed under the Creative Commons Attribution License, which permits unrestricted use, distribution, and reproduction in any medium, provided the original work is properly cited.

\begin{abstract}
We apply the Fourier-least squares method (FLSM) which allows us to find approximate periodic solutions for a very general class of nonlinear differential equations modelling oscillatory phenomena. We illustrate the accuracy of the method by using several significant examples of nonlinear problems including the cubic Duffing oscillator, the Van der Pol oscillator, and the Jerk equations. The results are compared to those obtained by other methods.
\end{abstract}

\section{Introduction}

Oscillatory phenomena are frequently encountered in various fields of science such as, for example, physics, molecular biology, and many branches of engineering. These oscillatory phenomena can be modelled using nonlinear differential equations. Nonlinear differential equations are one of the most important mathematical tools required for understanding these oscillatory phenomena present in everyday life. As is known, finding exact solutions of nonlinear differential equations is possible only in some particular cases. This justifies the need to resort to approximate methods for the computation of approximate periodic solutions, which in turn could provide important information about the phenomena studied.

In the present paper we apply the Fourier-least squares method (FLSM) for the computation of approximate periodic solutions for oscillatory phenomena modelled by nonlinear differential equations of the type

$$
x^{(n)}(t)=F\left(x^{(n-1)}(t), x^{(n-2)}(t), \ldots, x^{(1)}(t), x(t), t\right)
$$

with the initial conditions

$$
x^{(k)}(0)=A_{k}, \quad k \in 0,1,2, \ldots, n-1,
$$

where $F$ is a nonlinear continuous function, $t \in \mathbb{R}, A_{k} \in \mathbb{R}$.
Equation (1) is a very general one, being able to model a large class of oscillatory phenomena. As a consequence, since our method can be applied for (1), it follows that it can be considered a powerful and useful method.

We remark that recently there has been much interest in finding approximate periodic solutions of nonlinear differential equations of type (1) and conditions (2) and many approximate methods were proposed for the computation of approximate periodic solutions of equations of this type.

Among the methods used to compute such approximate periodic solutions we mention the following.

The homotopy perturbation method (see, $[1-5]$ ), the variational formulation method (see, [5-7]), the harmonic balance methods (see, [8-13]), the quasilinearization technique (see, [14]), the reproducing kernel space method (see, [15]), the Adomian decomposition method (see, [16]), the parameter-expansion method (see, $[17,18]$ ), the variational iteration methods (see, [19-21]), the energy balance method (see, $[20,22]$ ), the amplitude-frequency formulation (see, [4, 18]), the homotopy analysis method (see, [23-25]), the maxmin approach (see, [18]), the optimal homotopy asymptotic method (see, [26]), the residue harmonic balance method (see, [27]), the enhanced cubication method (see, [28]), the linearisation method (see, [29]), perturbation methods (see, [30]), and numerical methods (see, [31-35]).

If the problem consisting of (1) and conditions (2) admits a periodic solution, FLSM allows us to determine 
an accurate approximate solution of this problem. In order to test the accuracy of the method, we apply it to several well-known examples of nonlinear equations and compare the approximate solutions obtained with this method with approximate solutions obtained by other methods.

\section{The Fourier-Least Squares Method}

We suppose that the problem consisting of (1) and conditions (2) admit a periodic solution with the period $T$ and corresponding frequency $\omega=(2 \cdot \pi) / T$. We consider the operator

$$
\begin{aligned}
D & (x) \\
& =x^{(n)}(t)-F\left(x^{(n-1)}(t), x^{(n-2)}(t), \ldots, x^{(1)}(t), x(t), t\right) .
\end{aligned}
$$

If $\tilde{x}$ is an approximate periodic solution of (1), we evaluate the error obtained by replacing the exact solution $x$ with the approximate one $\tilde{x}$ as the remainder:

$$
R(t, \tilde{x})=D(\tilde{x}(t)), \quad t \in \mathbb{R} .
$$

Definition 1. One calls an Fourier-function a function of the form

$$
f(t)=\sum_{k=0}^{m}\left[a_{k} \cdot \cos (k \cdot \omega \cdot t)+b_{k} \cdot \sin (k \cdot \omega \cdot t)\right],
$$

where $a_{k}, b_{k}, \omega \in \mathbb{R}, m \in \mathbb{N}$.

We will find approximate Fourier-solutions $\tilde{x}$ of the problem consisting of (1) and conditions (2) on $\mathbb{R}$ which satisfy the following conditions:

$$
\begin{gathered}
|R(t, \tilde{x})|<\epsilon, \\
\tilde{x}(0)=A_{0}, \quad \tilde{x}^{(1)}(0)=A_{1},
\end{gathered}
$$

where

$$
\tilde{x}=\sum_{k=0}^{p}\left[\tilde{a}_{k} \cdot \cos (k \cdot \widetilde{\omega} \cdot t)+\tilde{b}_{k} \cdot \sin (k \cdot \tilde{\omega} \cdot t)\right] .
$$

Definition 2. One calls a $\epsilon$-approximate Fourier-solution of the problem consisting of (1) and conditions (2) a Fourierfunction $\tilde{x}$ satisfying the relations (6) and (7).

Definition 3. One calls a weak $\delta$-approximate Fourier-solution of the problem consisting of (1) and conditions (2) a Fourierfunction $\tilde{x}$ satisfying the relation

$$
\int_{0}^{2 \pi / \widetilde{\omega}} R^{2}(t, \tilde{x}) d t \leq \delta
$$

together with the initial conditions (7).

Definition 4. One calls a Fourier-sequence a sequence of Fourier-functions $\left\{s_{p}(t)\right\}_{p \in \mathbb{N}}$ :

$$
s_{p}(t)=\sum_{k=0}^{p}\left[a_{p k} \cdot \cos \left(k \cdot \omega_{p} \cdot t\right)+b_{p k} \cdot \sin \left(k \cdot \omega_{p} \cdot t\right)\right],
$$

where $a_{p k}, b_{p k} \in \mathbb{R}, b_{p 0}=0$.
Definition 5. One considers a Fourier-sequence satisfying the conditions

$$
s_{p}(0)=A_{0}, \quad s_{p}^{(1)}(0)=A_{1} .
$$

We call the Fourier-sequence $\left\{s_{p}(t)\right\}_{p \in \mathbb{N}}$ convergent to the solution of the problem consisting of (1) and conditions (2) if

$$
\lim _{p \rightarrow \infty} R\left(t, s_{p}(t)\right)=0
$$

We will find a weak $\epsilon$-approximate Fourier-solution of the type (8), where $p \geq 1$ and the constants $\widetilde{a}_{k}, \widetilde{b}_{k}, \widetilde{\omega}$ are calculated using the following steps.

(i) By substituting the approximate solution (8) in (1) we obtain the following expression:

$$
\begin{aligned}
& \Re\left(t, \tilde{a}_{0}, \tilde{a}_{1}, \ldots, \tilde{a}_{p}, \widetilde{b}_{1}, \ldots, \tilde{b}_{p}, \widetilde{\omega}\right) \\
&=R(t, \tilde{x})= \widetilde{x}^{(n)}(t) \\
& \quad-F\left(\tilde{x}^{(n-1)}(t), \tilde{x}^{(n-2)}(t), \ldots, \tilde{x}^{(1)}(t), \tilde{x}(t), t\right) .
\end{aligned}
$$

(ii) We attach to the problem consisting of (1) and conditions (2) the following real functional:

$$
\begin{aligned}
& J\left(\tilde{a}_{1}, \ldots, \tilde{a}_{p}, \tilde{b}_{2}, \ldots, \tilde{b}_{p}, \widetilde{\omega}\right) \\
& \quad=\int_{0}^{2 \pi / \widetilde{\omega}} \Re^{2}\left(t, \widetilde{a}_{0}, \tilde{a}_{1}, \ldots, \widetilde{a}_{p}, \widetilde{b}_{1}, \ldots, \widetilde{b}_{p}, \widetilde{\omega}\right) d t
\end{aligned}
$$

where $\tilde{a}_{0}, \widetilde{b}_{1}$ are computed as functions of $\tilde{a}_{1}, \ldots, \tilde{a}_{p}$, $\tilde{b}_{2}, \ldots, \widetilde{b}_{p}$ by using the initial conditions (7).

(iii) We compute the values of $a_{1}^{0}, \ldots, a_{p}^{0}, b_{2}^{0}, \ldots, b_{p}^{0}, \omega^{0}$ as the values which give the minimum of the functional (14) and the values of $a_{0}^{0}, b_{1}^{0}$ again as functions of $a_{1}^{0}, \ldots, a_{p}^{0}, b_{2}^{0}, \ldots, b_{p}^{0}$ by using the initial conditions (7).

(iv) Using the constants $a_{0}^{0}, a_{1}^{0}, \ldots, a_{p}^{0}, b_{1}^{0}, \ldots, b_{p}^{0}, \omega^{0}$ thus determined, we consider the Fourier-sequence

$$
\begin{gathered}
s_{p}(t)=\sum_{k=0}^{p}\left[a_{p k} \cdot \cos \left(k \cdot \omega_{p} \cdot t\right)+b_{p k} \cdot \sin \left(k \cdot \omega_{p} \cdot t\right)\right] \\
\text { with } a_{p k}=a_{k}^{0} \text { for } k=0, \ldots, p, b_{p k}=b_{k}^{0} \text { for } k= \\
1, \ldots, p \text {, and } \omega_{p}=\omega^{0}\left(\text { where } b_{0}^{0}=0\right) .
\end{gathered}
$$

The following convergence theorem holds.

Theorem 6. If the problem consisting of (1) and conditions (2) admits a periodic solution, then the Fourier-sequence $s_{p}(t)$ from (15) satisfies the property

$$
\lim _{p \rightarrow \infty} \int_{0}^{2 \pi / \omega_{p}} R^{2}\left(t, s_{p}(t)\right) d t=0 .
$$


Moreover, for all $\epsilon>0, \exists p_{0} \in \mathbb{N}$ such that for all $p \in \mathbb{N}, p>p_{0}$ it follows that $s_{p}(t)$ is a weak $\epsilon$-approximate Fourier-solution of the problem consisting of (1) and conditions (2).

Proof. From the fact that the problem consisting of (1) and conditions (2) admits a periodic solution it follows that the series

$$
\sum_{k=0}^{\infty}\left[a_{k} \cdot \cos (k \cdot \omega \cdot t)+b_{k} \cdot \sin (k \cdot \omega \cdot t)\right]
$$

exists and its sequence of partial sums $f_{p}$

$$
f_{p}(t)=\sum_{k=0}^{p}\left[a_{k} \cdot \cos (k \cdot \omega \cdot t)+b_{k} \cdot \sin (k \cdot \omega \cdot t)\right]
$$

converges to the solution of the problem consisting of (1) and conditions (2); that is,

$$
\lim _{p \rightarrow \infty} R\left(t, f_{p}(t)\right)=0 .
$$

Based on the way the Fourier-function $s_{p}(t)$ is computed and taking into account the relations (8)-(15), the following inequality holds:

$$
\begin{aligned}
0 & \leq \int_{0}^{2 \pi / \omega_{p}} R^{2}\left(t, s_{p}(t)\right) d t \\
& \leq \int_{0}^{2 \pi / \omega_{p}} R^{2}\left(t, f_{p}(t)\right) d t, \quad \forall p \in \mathbb{N} .
\end{aligned}
$$

It follows that

$$
\begin{aligned}
0 & \leq \lim _{p \rightarrow \infty} \int_{0}^{2 \pi / \omega_{p}} R^{2}\left(t, s_{p}(t)\right) d t \\
& \leq \lim _{p \rightarrow \infty} \int_{0}^{2 \pi / \omega_{p}} R^{2}\left(t, f_{p}(t)\right) d t=0, \quad \forall p \in \mathbb{N} .
\end{aligned}
$$

We obtain

$$
\lim _{p \rightarrow \infty} \int_{0}^{2 \pi / \omega_{p}} R^{2}\left(t, s_{p}(t)\right) d t=0 .
$$

From this limit we obtain that for all $\epsilon>0, \exists p_{0} \in \mathbb{N}$ such that for all $p \in \mathbb{N}, p>p_{0}$ it follows that $s_{p}(t)$ is a weak $\epsilon$ approximate Fourier-solution of the problem consisting of (1) and conditions (2).

Remark 7. Any $\epsilon$-approximate Fourier-solution of the problem consisting of (1) and conditions (2) is also a weak approximate Fourier-solution, but the opposite is not always true. It follows that the set of weak approximate Fouriersolutions of the problem consisting of (1) and conditions (2) also contains the approximate Fourier-solutions of the problem.

Taking into account the above remark, in order to find $\epsilon$ approximate Fourier-solutions of the problem consisting of (1) and conditions (2) by the Fourier-Least Squares Method, we will first determine weak approximate Fourier-solutions, $\tilde{x}$. If $|R(t, \tilde{x})|<\epsilon$, then $\tilde{x}$ is also an $\epsilon$-approximate Fouriersolution of the problem.

\section{Applications}

The test problems included this section are the Duffing oscillator (two cases, an autonomous one and one involving integral forcing terms) and the Jerk equations.

These problems were extensively studied over the years, and various solutions, both approximate analytical ones and numerical ones, were proposed.

The qualitative properties of these oscillators were also extensively studied. Stability and bifurcation studies for the Duffing oscillators include [36-38] among many others. For Jerk-type equations a comprehensive bifurcation study can be found in [39] and a study of the limit cycles can be found in [40]. Following the computations presented in these papers, corresponding conclusions can be drawn for the problems studied in the Sections 3.1-3.3. For example, in the case of the autonomous Duffing oscillator (23), for the values of the parameters considered in the computations $(\epsilon=1.25$ and $A=2$ ), a quick computation similar to the one in [36] indicates that the only equilibrium point is the origin, which is a center; similar computations can be performed for the other problems.

In the following we compute approximate solutions for the Duffing oscillator and the Jerk equations and compare our results with similar analytical approximations previously computed by using other methods.

3.1. Application 1: The Autonomous Duffing Oscillator. Our first test problem is the autonomous Duffing oscillator:

$$
\begin{gathered}
x^{(2)}(t)+x(t)+\epsilon \cdot x^{3}(t)=0, \\
x(0)=A, \quad x^{(1)}(0)=0 .
\end{gathered}
$$

The Duffing oscillator is extensively studied in literature and some relatively recent results are presented in $[12,19]$. In [12] approximate solutions are computed using the rational harmonic balance method (RHB) and in [19] approximate solutions of (23) are computed using a variational iteration procedure (VI). The approximate frequency of the oscillations obtained by using these methods is compared with the exact period known in the literature.

In the following, in order to obtain our approximation of the frequency and of the solution, we will perform the steps described in the previous section. The computations were performed using the SAGE open source software (version 5.5, available at http://www.sagemath.org/).

We will perform in detail our computations for the values $\epsilon=1.25$ and $A=2$.

Since the computations of the minimum of the functional (14) are relatively difficult for large values of $p$ in (8), we will actually use an iterative procedure, starting with $p=1$ and increasing the value until we achieve the desired precision.

3.1.1. Approximate Solution for $p=1$. Taking into account these considerations, first we choose the approximate solution (8) of the form

$$
\tilde{x}_{1}(t)=\widetilde{a}_{0}+\tilde{a}_{1} \cdot \cos \left(\widetilde{\omega}_{1} \cdot t\right)+\widetilde{b}_{1} \cdot \sin \left(\widetilde{\omega}_{1} \cdot t\right) .
$$


In Step 1, the expression (13) becomes

$$
\begin{aligned}
\Re( & \left.t, \tilde{a}_{0}, \tilde{a}_{1}, b_{1}, \widetilde{\omega}_{1}\right) \\
= & \widetilde{a}_{1} \widetilde{\omega}_{1}^{2} \cos \left(\widetilde{\omega}_{1} \cdot t\right)-\widetilde{b}_{1} \widetilde{\omega}_{1}^{2} \sin \left(\widetilde{\omega}_{1} \cdot t\right) \\
& +\frac{5}{4}\left(\widetilde{a}_{1} \cos \left(\widetilde{\omega}_{1} \cdot t\right)+\widetilde{b}_{1} \sin \left(\widetilde{\omega}_{1} \cdot t\right)+\widetilde{a}_{0}\right)^{3} \\
& +\widetilde{a}_{1} \cos \left(\widetilde{\omega}_{1} \cdot t\right)+\widetilde{b}_{1} \sin \left(\widetilde{\omega}_{1} \cdot t\right)+\widetilde{a}_{0} .
\end{aligned}
$$

Taking into account the initial conditions $\tilde{x}(0)=A$, $\tilde{x}^{(1)}(t)=0$ we obtain the relations

$$
\tilde{a}_{0}=-\widetilde{a}_{1}+2, \quad \tilde{b}_{1}=0 .
$$

Replacing these values, the corresponding functional (14) from Step 2 is

$$
\begin{aligned}
J\left(\widetilde{a}_{1}, \widetilde{\omega}_{1}\right)=( & 36864 \pi+5775 \pi \widetilde{a}_{1}^{6}+128 \pi \widetilde{a}_{1}^{2} \widetilde{\omega}_{1}^{4} \\
& \left.-37800 \pi \widetilde{a}_{1}^{5}+107800 \pi \widetilde{a}_{1}^{4}\right) \cdot \frac{1}{128 \widetilde{\omega}_{1}} \\
+ & \left(-172800 \pi \widetilde{a}_{1}^{3}-16\right. \\
& \times\left(75 \pi \widetilde{a}_{1}^{4}-240 \pi \widetilde{a}_{1}^{3}+256 \pi \widetilde{a}_{1}^{2}\right) \widetilde{\omega}_{1}^{2} \\
& \left.+167424 \pi \widetilde{a}_{1}^{2}-98304 \pi \widetilde{a}_{1}\right) \cdot \frac{1}{128 \widetilde{\omega}_{1}} .
\end{aligned}
$$

In Step 3 we must compute the minimum of $J$ with respect to $\tilde{a}_{1}$ and $\widetilde{\omega}_{1}$. For relatively simple problems such as this it is possible to compute directly the critical points of $J$ and subsequently select the value corresponding to the minimum.

In general, the critical points corresponding to the functional $(14) J\left(\widetilde{a}_{1}, \ldots, \tilde{a}_{p}, \widetilde{b}_{2}, \ldots, \tilde{b}_{p}, \widetilde{w}\right)$ are the solution of the system:

$$
\begin{gathered}
\frac{\partial J}{\partial \widetilde{a}_{1}}=0, \\
\vdots \\
\frac{\partial J}{\partial \widetilde{a}_{p}}=0, \\
\frac{\partial J}{\partial \widetilde{b}_{2}}=0, \\
\vdots \\
\frac{\partial J}{\partial \widetilde{b}_{p}}=0, \\
\frac{\partial J}{\partial \widetilde{\omega}_{1}}=0 .
\end{gathered}
$$

For $p=1$ the system becomes

$$
\begin{aligned}
& -\left(49152 \pi-17325 \pi \widetilde{a}_{1}^{5}-128 \pi \widetilde{a}_{1} \widetilde{\omega}^{4}\right. \\
& \left.+94500 \pi \widetilde{a}_{1}^{4}-215600 \pi \widetilde{a}_{1}^{3}\right) \cdot \frac{1}{64 \widetilde{\omega}_{1}} \\
& +\left(32\left(75 \pi \widetilde{a}_{1}^{3}-180 \pi \widetilde{a}_{1}^{2}+128 \pi \widetilde{a}_{1}\right) \widetilde{\omega}^{2}\right. \\
& \left.+259200 \pi \widetilde{a}_{1}^{2}-167424 \pi \widetilde{a}_{1}\right) \cdot \frac{1}{64 \widetilde{\omega}_{1}}=0, \\
& \left(16 \pi \widetilde{a}_{1}^{2} w^{3}-\left(75 \pi \widetilde{a}_{1}^{4}-240 \pi \widetilde{a}_{1}^{3}+256 \pi \widetilde{a}_{1}^{2}\right) \widetilde{\omega}\right) \cdot \frac{1}{4 \widetilde{\omega}_{1}} \\
& -\left(36864 \pi+5775 \pi \widetilde{a}_{1}^{6}+128 \pi \widetilde{a}_{1}^{2} \widetilde{\omega}^{4}-37800 \pi \widetilde{a}_{1}^{5}\right. \\
& \left.+107800 \pi \widetilde{a}_{1}^{4}-172800 \pi \widetilde{a}_{1}^{3}\right) \cdot \frac{1}{128 \widetilde{\omega}_{1}^{2}} \\
& +\left(-16\left(75 \pi \widetilde{a}_{1}^{4}-240 \pi \widetilde{a}_{1}^{3}+256 \pi \widetilde{a}_{1}^{2}\right) \widetilde{\omega}^{2}\right. \\
& \left.+167424 \pi \widetilde{a}_{1}^{2}-98304 \pi \widetilde{a}_{1}\right) \cdot \frac{1}{128 \widetilde{\omega}_{1}^{2}}=0 .
\end{aligned}
$$

This system can be solved directly. We used the "solve" command in SAGE and, after we excluded the complex solutions, we found the critical points

$$
\begin{array}{cc}
\widetilde{a}_{1}=1.95861253449, & \widetilde{\omega}_{1}=2.16432748538, \\
\widetilde{a}_{1}=1.95861253449, & \widetilde{\omega}_{1}=-2.16432748538, \\
\widetilde{a}_{1}=0.181528334876, & \widetilde{\omega}_{1}=-6.92142088267, \\
\widetilde{a}_{1}=0.181528334876, & \widetilde{\omega}_{1}=6.92142088267 .
\end{array}
$$

In order to find the minimum, we use the second partial derivative test, which is easy enough to implement in SAGE, and find that $\widetilde{a}_{1}=a_{1}^{0}=1.95861253449, \widetilde{\omega}_{1}=\omega_{1}^{0}=$ 2.16432748538 is the local minima. Using again the relations $a_{0}^{0}=-a_{1}^{0}+2, b_{1}^{0}=0$, we replace all the values in the expression of the approximate solution (8) and we obtain our first approximation:

$$
\begin{aligned}
\tilde{x}_{1}(t)= & 1.95861253449 \cos (2.16432748538 t) \\
& +0.0413874655104
\end{aligned}
$$

In Figure 1 we present the comparison between our $\tilde{x}_{1}$ approximate solution (solid line) and the numerical solution obtained by using a fourth order Runge-Kutta method (dotted line).

As we already saw, the approximate value of the frequency obtained here is $\widetilde{\omega}_{1}=\omega_{1}^{0}=2.16432748538$ and it is already close to the exact value which in this case is $\omega_{\text {ex }}=$ 2.150416169536 .

We observe that, generally speaking, if the value of $p$ in the expression of $\tilde{x}(t)$ is larger than 1 , then the solution of the corresponding system of equations which gives the critical 


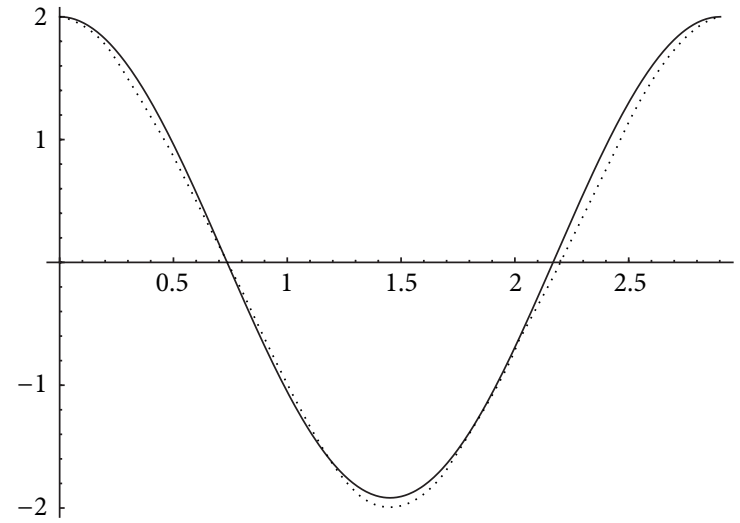

Figure 1: Comparison between $\tilde{x}_{1}$ (solid line) and numerical solution (dotted line).

points (28) cannot be found directly. In the particular case of SAGE, the command "solve" fails to find the solutions, exiting with some kind of error message.

In this situation it is still possible to find good approximations of the solutions of the problem solving the system (28) by means of a numerical method. More precisely, we can find approximate solutions for the given problem (23) solving (28) by means of a SAGE implementation of the well-known Newton method.

3.1.2. Approximate Solution for $p>1$. As the following results will show, the Newton method is able to find approximate solutions of (28) which can lead to highly accurate approximate solutions of the problem (23).

For $p=2$ the approximate solution (8) has the form

$$
\begin{aligned}
\widetilde{x}_{2}(t)= & \widetilde{a}_{0}+\widetilde{a}_{1} \cdot \cos \left(\widetilde{\omega}_{2} \cdot t\right) \\
& +\widetilde{b}_{1} \cdot \sin \left(\widetilde{\omega}_{2} \cdot t\right)+\widetilde{a}_{2} \cdot \cos \left(2 \cdot \widetilde{\omega}_{2} \cdot t\right) \\
& +\widetilde{b}_{2} \cdot \sin \left(2 \cdot \widetilde{\omega}_{2} \cdot t\right) .
\end{aligned}
$$

After we compute the corresponding expressions of $\Re\left(t, \widetilde{a}_{0}, \widetilde{a}_{1}, \widetilde{a}_{2}, \widetilde{b}_{1}, \widetilde{b}_{2}, \widetilde{\omega}_{2}\right)$ and $J\left(\widetilde{a}_{1}, \widetilde{a}_{2}, \widetilde{b}_{2}, \widetilde{\omega}_{2}\right)$ and of the system (28) (all too large to insert here), we apply Newton's method taking as the starting point of the iteration $\left(\widetilde{a}_{1, s}, \widetilde{a}_{2, s}, \widetilde{b}_{2, s}, \widetilde{\omega}_{2, s}\right)$, where $\widetilde{a}_{1, s}$ and $\widetilde{\omega}_{2, s}$ are the values of $a_{1}^{0}$ and $\omega_{1}^{0}$ computed for the previous approximation $p=1$, namely, $\widetilde{a}_{1, s}=$ 1.95861253449 and $\widetilde{\omega}_{2, s}=2.16432748538$. In order for the sequence of approximations given by Newton's method to converge to the solution(s) of the system (28), $\widetilde{a}_{2, s}$ and $\widetilde{b}_{2, s}$ will take successively values on a given grid of the type $G=$ $I_{\widetilde{a}_{2}} \times I_{\tilde{b}_{2}}$, where $I_{i}$ is a division of a symmetric interval centered in zero.

For all the test problems included in this paper and for all the values of $p$ tested, a grid of the form $G=$ $\{-1,0.9,-0.8, \ldots, 1\} \times\{-1,0.9,-0.8, \ldots, 1\}$ (i.e., from -1 to 1 with step size 0.1 ) is large enough in the sense that if the starting point $\left(s_{0}, s_{1}\right)$ scans $G$ we can obtain using Newton's method the desired solutions of (28).

In the particular case of the problem (23) it was actually sufficient to choose $\widetilde{a}_{2, s}=0$ and $\widetilde{b}_{2, s}=0$ and the sequence converged to the minimum of $J$.

For $p=3$ we repeat the same procedure: we compute the corresponding expressions of $\Re\left(t, \widetilde{a}_{0}, \widetilde{a}_{1}, \widetilde{a}_{2}, \widetilde{a}_{3}, \widetilde{b}_{1}, \widetilde{b}_{2}, \widetilde{b}_{3}, \widetilde{\omega}_{3}\right)$ and $J\left(\widetilde{a}_{1}, \widetilde{a}_{2}, \widetilde{a}_{3}, \widetilde{b}_{2}, \widetilde{b}_{3}, \widetilde{\omega}_{3}\right)$ and of the system (28) and we apply Newton's method taking as the starting point of the iteration $\left(\widetilde{a}_{1, s}, \widetilde{a}_{2, s}, \widetilde{a}_{3, s}, \widetilde{b}_{2, s}, \widetilde{b}_{3, s}, \widetilde{\omega}_{3, s}\right)$, where $\left(\widetilde{a}_{1, s}, \widetilde{a}_{2, s}, \widetilde{b}_{3, s}, \widetilde{\omega}_{3, s}\right)$ are the minimum values $\left(a_{1}^{0}, a_{2}^{0}, b_{2}^{0}, \omega_{2}^{0}\right)$ computed for the previous approximation $p=2$ and $a_{3, s}=b_{3, s}=0$.

The process can be carried on for increased values of $p$ until the desired accuracy is reached.

While at a first look the computations may seem long and tedious, by using a software program such as SAGE, we were actually able to perform them easy and quick, obtaining a very good accuracy. Thus, for $p=7$, we obtained for the approximate frequency the value $\omega_{7}^{0}=2.15041853722$ and the corresponding approximate solution (8) has the form

$$
\begin{aligned}
\tilde{x}_{7}(t)= & 1.0618674103207715 \cdot 10^{-08} \\
& +1.930547907025286 \\
& \cdot \cos (2.150418537217718 \cdot t) \\
& +1.5538232860698776 \cdot 10^{-08} \\
& \cdot \cos (4.300837074435436 \cdot t) \\
& +0.0671185574473875 \\
& \cdot \cos (6.4512556116531545 \cdot t) \\
& +1.4239017145731592 \cdot 10^{-09} \\
& \cdot \cos (8.601674148870872 \cdot t) \\
& +0.0022576839638690305 \\
& \cdot \cos (10.75209268608859 \cdot t) \\
& +1.3333451751464932 \cdot 10^{-10} \\
& \cdot \cos (12.902511223306309 \cdot t) \\
& +7.582384931426247 \cdot 10^{-05} \\
& \cdot \cos (15.052929760524027 \cdot t) .
\end{aligned}
$$

In Figure 2 we present the comparison between the $\tilde{x}_{7}$ approximate solution (solid line) and the numerical solution obtained by using a fourth order Runge-Kutta method (dotted line).

In [19] approximate solutions for the problem (23) were computed for the cases $\epsilon=1.25, A=2$ (studied above) and $\epsilon=250, A=2$. 


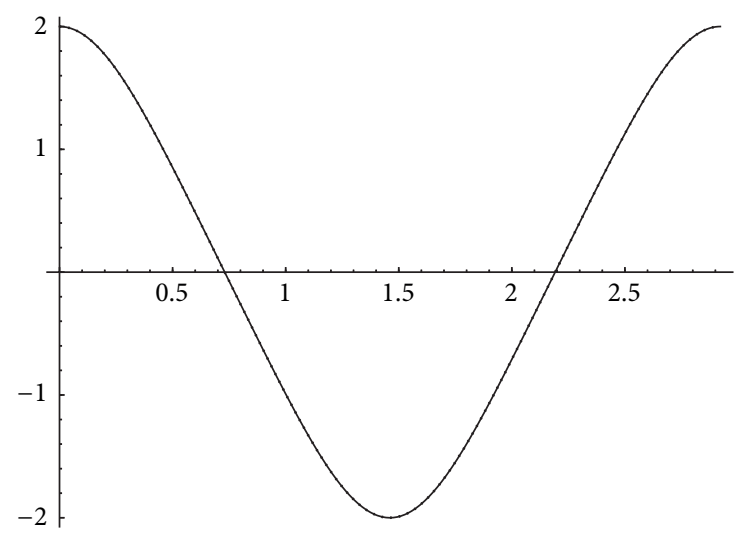

Figure 2: Comparison between $\tilde{x}_{7}$ (solid line) and numerical solution (dotted line).

TABLE 1: Comparison of the absolute errors of the approximate solutions for problem (23) in the case $\epsilon=1.25, A=2$.

\begin{tabular}{lcc}
\hline & \multicolumn{1}{c}{$x_{\mathrm{VI}}$} & $\tilde{x}_{7}$ \\
\hline$t=0$ & $5.34289999998 \cdot 10^{-5}$ & 0.00000000000 \\
$t=0.4$ & $8.81935946956 \cdot 10^{-2}$ & $3.92745813649 \cdot 10^{-7}$ \\
$t=0.8$ & $3.40733004552 \cdot 10^{-2}$ & $1.66866686269 \cdot 10^{-6}$ \\
$t=1.2$ & $5.63129918651 \cdot 10^{-2}$ & $5.23059939095 \cdot 10^{-6}$ \\
$t=1.6$ & $1.96411171977 \cdot 10^{-2}$ & $1.89375331883 \cdot 10^{-6}$ \\
$t=2$ & $7.93642203903 \cdot 10^{-2}$ & $6.81658032309 \cdot 10^{-6}$ \\
$t=2.4$ & $8.23170554343 \cdot 10^{-2}$ & $1.36265840209 \cdot 10^{-5}$ \\
$t=2.8$ & $1.50931697081 \cdot 10^{-2}$ & $9.46274851943 \cdot 10^{-6}$ \\
\hline
\end{tabular}

For the case $\epsilon=1.25, A=2$, the approximate solution from [19] is

$$
\begin{aligned}
x_{\mathrm{VI}}(t)= & 1.989351555 \cdot \cos (2.15031 \cdot t) \\
& +0.008334403 \cdot \cos (6.45093 \cdot t) \\
& +0.002206664 \cdot \cos (10.75155 \cdot t) \\
& +0.000053689 \cdot \cos (15.05217 \cdot t) \\
& +\left(2.6 \cdot 10^{-7}\right) \cdot \cos (19.35279 \cdot t) .
\end{aligned}
$$

Table 1 presents the comparison of the absolute errors (computed as the difference in absolute value between the approximate solution and the corresponding numerical solution given by the Runge-Kutta method) corresponding to the approximate solutions $x_{\mathrm{VI}}$ (see, [19]) and $\tilde{x}_{7}$ for the case $\epsilon=1.25, A=2$.

For the case $\epsilon=250, A=2$, the approximate solution from [19] is

$$
\begin{aligned}
x_{\mathrm{VI}}(t)= & 1.977507645 \cdot \cos (26.8026 \cdot t) \\
& +0.018844215 \cdot \cos (80.4078 \cdot t)
\end{aligned}
$$

TABLE 2: Comparison of the absolute errors of the approximate solutions for problem (23) in the case $\epsilon=250, A=2$.

\begin{tabular}{lcc}
\hline & \multicolumn{1}{c}{$x_{\mathrm{VI}}$} & $\tilde{x}_{7}$ \\
\hline$t=0$ & $1.63917000000 \cdot 10^{-4}$ & 0.00000000000 \\
$t=0.04$ & $9.94430509655 \cdot 10^{-2}$ & $1.59432943392 \cdot 10^{-6}$ \\
$t=0.08$ & $1.01602511979 \cdot 10^{-1}$ & $2.50754265524 \cdot 10^{-5}$ \\
$t=0.12$ & $1.84880609089 \cdot 10^{-3}$ & $1.44364392085 \cdot 10^{-6}$ \\
$t=0.16$ & $9.38684852713 \cdot 10^{-2}$ & $2.52297827337 \cdot 10^{-5}$ \\
$t=0.2$ & $1.00379880594 \cdot 10^{-1}$ & $4.54516346458 \cdot 10^{-5}$ \\
\hline
\end{tabular}

$$
\begin{aligned}
& +0.003619986 \cdot \cos (134.013 \cdot t) \\
& +0.000027438 \cdot \cos (187.6182 \cdot t) \\
& +0.000164633 \cdot \cos (241.2234 \cdot t)
\end{aligned}
$$

For this case, the approximate solution computed using our method is

$$
\begin{aligned}
\tilde{x}_{7}(t)= & 3.22758664474 \cdot 10^{-08}+1.91015330733 \\
& \cdot \cos (26.810846231 \cdot t)+\left(6.9616769357 \cdot 10^{-08}\right) \\
& \cdot \cos (53.6216924619 \cdot t)+0.0859766881274 \\
& \cdot \cos (80.4325386929 \cdot t)+\left(7.78739552572 \cdot 10^{-09}\right) \\
& \cdot \cos (107.243384924 \cdot t)+0.00371019879647 \\
& \cdot \cos (134.054231155 \cdot t)+\left(7.82719602479 \cdot 10^{-10}\right) \\
& \cdot \cos (160.865077386 \cdot t)+0.0001596952849 \\
& \cdot \cos (187.675923617 \cdot t)
\end{aligned}
$$

Table 2 presents the comparison of the absolute errors (computed as the difference in absolute value between the approximate solution and the corresponding numerical solution given by the Runge-Kutta method) corresponding to the approximate solutions $x_{\mathrm{VI}}$ (see, [19]) and $\tilde{x}_{7}$ for the case $\epsilon=250, A=2$.

Finally, in Table 3, we compare the approximate values for the frequency $\omega_{7}^{0}$ computed using our method with approximate values computed in [19] $\left(\omega_{\mathrm{VI}}\right)$ and [12] $\left(\omega_{\mathrm{RHB}}\right)$. The comparison is made by means of the percentage error, which for a given approximate error $\omega_{\text {approx }}$ is defined as $e_{\omega_{\text {approx }}}=100 \cdot\left(\left|\omega_{\text {exact }}-\omega_{\text {approx }}\right| / \omega_{\text {exact }}\right)$, where $\omega_{\text {exact }}$ is the corresponding exact error. It is easy to see that our approximations are far better than the ones previously computed and they remain accurate even for the case of a very strong nonlinearity. 
TABLE 3: Comparison of approximate errors for the frequency $\omega$ for problem (23).

\begin{tabular}{lcccc}
\hline$\epsilon \cdot A^{2}$ & 5 & 100 & 1000 & 10000 \\
$\omega_{\text {exact }}$ & 2.1504161695 & 8.5335861889 & 26.8107384581 & 84.7274799361 \\
$\omega_{\mathrm{VI}}$ & 2.15031 & $\mathrm{x}$ & 26.8026 & 84.7015 \\
$e_{\omega_{\mathrm{VI}}}(\%)$ & 0.004937 & $\mathrm{x}$ & 0.0303552 & 0.0306629 \\
$\omega_{\mathrm{RHB}}$ & 2.15045 & 8.53402 & $\mathrm{x}$ & $\mathrm{x}$ \\
$e_{\omega_{\mathrm{RHB}}}(\%)$ & 0.0015 & 0.0051 & $\mathrm{x}$ & $\mathrm{x}$ \\
$\omega_{7}$ & 2.15041853722 & 8.53361825153 & 26.810846231 & 84.7278228489 \\
$e_{\omega_{7}}(\%)$ & 0.0001101034 & 0.0003757217 & 0.0004019763 & 0.0004047244 \\
\hline
\end{tabular}

3.2. Application 2: The Duffing Equation Involving Integral Forcing Terms. Our second test problem is

$$
\begin{gathered}
x^{(2)}(t)+x(t) \cdot x^{(1)}(t)+\int_{0}^{t} t s x^{2}(s) d s=f(t), \\
0<t<1, \\
x(0)-x^{(1)}(0)=0, \\
x(1)+x^{(1)}(1)=0,
\end{gathered}
$$

where $f(t)=-3 t-3 t^{2}+5 t^{3} / 2+2 t^{4} / 3-t^{5} / 4-2 t^{6} / 5+t^{7} / 6$.

The problem $(38)$ (see, $[14,15])$ is a version of the wellknown Duffing equation involving both integral and nonintegral forcing terms with separated boundary conditions. This equation has been studied in a series of recent papers including $[14,15]$.

In [14], the authors applied a generalized quasilinearization technique to prove the existence and uniqueness of the solution of Duffing equation involving both integral and nonintegral forcing terms. They showed that there are sequences of approximate solutions converging monotonically and quadratically to the unique solution of the problem.

In [15], the authors gave a representation of exact solution and approximate solution of Duffing equation involving both integral and nonintegral forcing terms in the reproducing kernel space (RKS). They represented the exact solution in the form of a series and they showed that the n-term approximation of the exact solution converges to the exact solution.

Next we present our results for (38) using FLSM. Also, we will compare these results with those obtained in [15].

Thus, for $p=3$, we obtained the fact that the approximate periodic solution (8) has the form

$$
\begin{aligned}
& \tilde{x}_{3}(t) \\
& =1.45453 \sin (t)-0.26409 \sin (2 t) \\
& \quad+0.0245502 \sin (3 t)+2.6625 \cos (t) \\
& \quad-0.169569 \cos (2 t)+0.00174065 \cos (3 t)-1.49467 .
\end{aligned}
$$

Since in [15] only the numerical results are presented while the expression of approximate solution expression is
TABLE 4: Comparison of the absolute errors of the approximate solutions for problem (38).

\begin{tabular}{lcc}
\hline & $x_{\mathrm{RKS}}$ & $\tilde{x}_{3}$ \\
\hline$t=0$ & 0 & 0 \\
$t=0.1$ & $9.46600 \cdot 10^{-6}$ & $9.85192 \cdot 10^{-8}$ \\
$t=0.2$ & $9.77708 \cdot 10^{-6}$ & $9.2974 \cdot 10^{-8}$ \\
$t=0.3$ & $9.81938 \cdot 10^{-6}$ & $1.58651 \cdot 10^{-7}$ \\
$t=0.4$ & $9.66575 \cdot 10^{-6}$ & $5.76015 \cdot 10^{-8}$ \\
$t=0.5$ & $9.37204 \cdot 10^{-6}$ & $2.07316 \cdot 10^{-7}$ \\
$t=0.6$ & $8.97794 \cdot 10^{-6}$ & $5.9859 \cdot 10^{-8}$ \\
$t=0.7$ & $8.50917 \cdot 10^{-6}$ & $1.55722 \cdot 10^{-7}$ \\
$t=0.8$ & $7.97984 \cdot 10^{-6}$ & $9.17107 \cdot 10^{-8}$ \\
$t=0.9$ & $7.39469 \cdot 10^{-6}$ & $9.65862 \cdot 10^{-8}$ \\
$t=1$ & $6.75105 \cdot 10^{-6}$ & $7.84328 \cdot 10^{-10}$ \\
\hline
\end{tabular}

not, we cannot perform a direct graphical comparison of our approximate solution with the corresponding solution from [15].

Therefore, in Table 4, we present the comparison of several values of the absolute errors (computed as the difference in absolute value between the exact solution and the approximate solution) corresponding to the approximate solutions $x_{\mathrm{RKS}}$ from [15] and to our approximate solutions for $t=0.1,0.2, \ldots, 1$, as given in [15].

3.3. Application 3: The Jerk Equation Containing VelocityCubed and Velocity Times Displacement-Squared. Our last test is a Jerk nonlinear equation, which describes several physical problems using mechanical oscillations of the third order. The most general form of the Jerk nonlinear equations, which contains the third temporal derivative of displacement, is

$$
\begin{aligned}
x^{(3)}+ & \alpha \cdot x \cdot x^{(1)} \cdot x^{(2)}+\beta \cdot x^{(1)} \cdot x^{(2)^{2}} \\
& +\delta \cdot x^{2} \cdot x^{(1)}+\varepsilon \cdot x^{(1)^{3}}+\gamma \cdot x^{(1)}=0,
\end{aligned}
$$

where the parameters $\alpha, \beta, \delta$, $\varepsilon$, and $\gamma$ are constants.

Nonlinear Jerk equations (40) are intensely studied by several authors in the literature and some recent results are presented in $[3,8,9,27]$. 


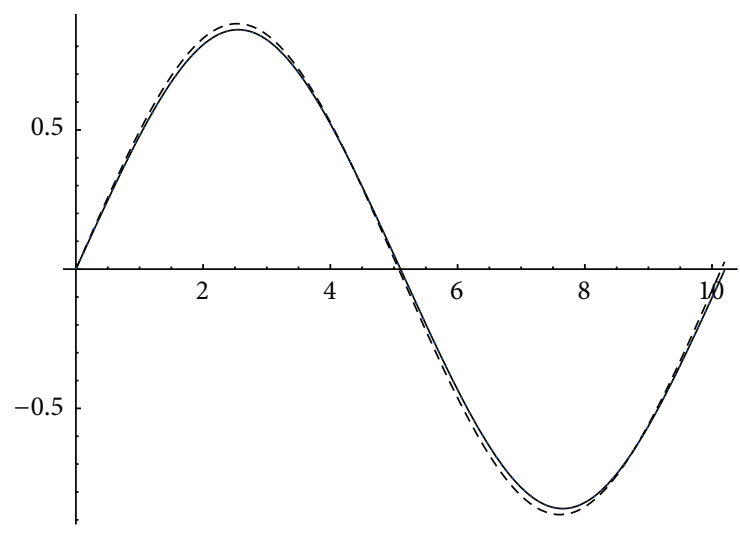

FIgURE 3: Comparison between our solution $\tilde{x}_{3}$ (solid line), solution $x_{\mathrm{HPM}}$ in [3] (dashed line), and numerical solution (dotted line).

We consider the following Jerk equation:

$$
\begin{gathered}
x^{(3)}+x^{2} \cdot x^{(1)}+x^{(1)^{3}}=0, \\
x(0)=0, \quad x^{(1)}(0)=B, \quad x^{(2)}(0)=0 .
\end{gathered}
$$

Recently, Ma et al. in [3], using the Homotopy Perturbation Method, obtained high-order analytic approximate periods and periodic solutions of the Jerk Equation (41). In [8,9], Gottlieb used the lowest-order harmonic balance method to determine analytical approximations to the periodic solution of the Jerk equations. Also, Leung and Guo in [27] obtained approximations for the angular frequency and the limit cycle for (41) based on the residue harmonic balance approach.

For $B=0.5$, the approximate periodic solution $x_{\mathrm{HPM}}$ from [3] is

$$
\begin{aligned}
x_{\mathrm{HPM}}(t)= & 0.8708027 \cdot \sin (0.61924 \cdot t)-0.0106486 \\
& \cdot \sin (1.85772 \cdot t)
\end{aligned}
$$

Applying FLSM we computed an approximate periodic solution of the problem (41) of the same order (containing terms up to $\sin (3 \cdot \omega \cdot t))$ :

$$
\begin{aligned}
\tilde{x}_{3}(t)= & 0.847907380098 \cdot \sin (0.615691681067 \cdot t) \\
& -\left(3.59275929188 \times 10^{-05}\right) \\
& \cdot \sin (1.23138336213 \cdot t) \\
& -0.0119135817912 \cdot \sin (1.8470750432 \cdot t) .
\end{aligned}
$$

The approximate frequency and period are $\omega_{3}^{0}=$ $0.615691681067, T_{3}^{0}=10.2050839736674$ with an error of $e_{\omega_{3}^{0}}=-0.0555984645279972$.

In Figure 3, we can visualize and compare our approximate solution (solid line), the approximate solution $x_{\mathrm{HPM}}$ from [3] (dashed line), and the numerical solution obtained by using a fourth order Runge-Kutta method (dotted line).

Table 5 presents the comparison of the absolute errors (computed as the difference in absolute value between the
TABLE 5: Comparison of the absolute errors corresponding to the solution $x_{\mathrm{HPM}}$ from [3] and to our solution $\tilde{x}_{3}$.

\begin{tabular}{lcc}
\hline & $x_{\mathrm{HPM}}$ & $\widetilde{x}_{3}$ \\
\hline$t=0$ & 0 & 0 \\
$t=1.0$ & $1.72354316028 \cdot 10^{-2}$ & $2.11130244099 \cdot 10^{-4}$ \\
$t=2.0$ & $2.3307287671 \cdot 10^{-2}$ & $1.31078376172 \cdot 10^{-4}$ \\
$t=3.0$ & $1.79768181549 \cdot 10^{-2}$ & $3.46325784747 \cdot 10^{-4}$ \\
$t=4.0$ & $4.72752471124 \cdot 10^{-3}$ & $8.73925906836 \cdot 10^{-4}$ \\
$t=5.0$ & $1.46107699703 \cdot 10^{-2}$ & $1.42164922819 \cdot 10^{-3}$ \\
$t=6.0$ & $3.05145610435 \cdot 10^{-2}$ & $1.51326363254 \cdot 10^{-3}$ \\
$t=7.0$ & $3.04290934289 \cdot 10^{-2}$ & $8.83072635498 \cdot 10^{-4}$ \\
$t=8.0$ & $1.45339383549 \cdot 10^{-2}$ & $7.00497125303 \cdot 10^{-4}$ \\
$t=9.0$ & $6.86925713324 \cdot 10^{-3}$ & $2.00167777983 \cdot 10^{-3}$ \\
$t=10.0$ & $2.91276307352 \cdot 10^{-2}$ & $2.84571545126 \cdot 10^{-3}$ \\
\hline
\end{tabular}

TABLE 6: Comparison of the absolute errors corresponding to the solution $x_{\mathrm{RHB}}$ from [27] and to our solution $\tilde{x}_{5}$.

\begin{tabular}{lcc}
\hline & $x_{\mathrm{RHB}}$ & $\tilde{x}_{5}$ \\
\hline$t=0$ & 0 & 0 \\
$t=1.0$ & $2.07336138332 \cdot 10^{-4}$ & $4.76705901847 \cdot 10^{-6}$ \\
$t=2.0$ & $8.18802985278 \cdot 10^{-4}$ & $7.01416299886 \cdot 10^{-6}$ \\
$t=3.0$ & $6.29650984827 \cdot 10^{-4}$ & $8.81003685005 \cdot 10^{-7}$ \\
$t=4.0$ & $3.27159888572 \cdot 10^{-4}$ & $1.19800236493 \cdot 10^{-5}$ \\
$t=5.0$ & $7.15072428261 \cdot 10^{-4}$ & $2.0505386494 \cdot 10^{-5}$ \\
$t=6.0$ & $7.95019646471 \cdot 10^{-4}$ & $2.11112229619 \cdot 10^{-5}$ \\
$t=7.0$ & $1.09803461819 \cdot 10^{-3}$ & $1.31470477691 \cdot 10^{-5}$ \\
$t=8.0$ & $5.35184978947 \cdot 10^{-4}$ & $5.37333608552 \cdot 10^{-6}$ \\
$t=9.0$ & $8.0197576264 \cdot 10^{-4}$ & $2.75784654626 \cdot 10^{-5}$ \\
$t=10.0$ & $1.42381620457 \cdot 10^{-3}$ & $3.9140208578 \cdot 10^{-5}$ \\
\hline
\end{tabular}

approximate solution and the corresponding numerical solution given by the Runge-Kutta method) corresponding to the approximate solution $x_{\mathrm{HPM}}$ from [3] and our approximate solution $\widetilde{x}_{3}$ for the case $B=0.5$.

Also, for $B=0.5$, the approximate periodic solution $x_{\mathrm{RHB}}$ from [27] is given by

$$
\begin{aligned}
x_{\mathrm{RHB}}(t)= & 0.8473604 \cdot \sin (0.6151734 \cdot t) \\
& -0.01168397 \cdot \sin (1.8455202 \cdot t) \\
& +0.000094103 \cdot \sin (3.075867 \cdot t) .
\end{aligned}
$$

Applying FLSM we computed an approximate periodic solution of the problem (41) of the same order (containing terms up to $\sin (5 \cdot \omega \cdot t))$ :

$$
\begin{aligned}
\tilde{x}_{5}(t)= & 0.847966627859 \\
& \cdot \sin (0.61535099365 \cdot t) \\
& +\left(2.70675259884 \cdot 10^{-08}\right)
\end{aligned}
$$


TABLE 7: Comparison of approximate periods.

\begin{tabular}{lcc}
\hline$B=0.5$ & Period & Error \\
\hline$T_{\text {exact }}$ & 10.210761 & 0 \\
FLSM $T_{1}$ & 10.1976548730449 & -0.128356025129356 \\
FLSM $T_{3}$ & 10.2050839736674 & -0.0555984645279972 \\
FLSM $T_{5}$ & 10.2107339908715 & -0.000264516312410058 \\
Wu et al. [41] $T_{2}$ & 10.1884 & -0.220 \\
Wu et al. [41] $T_{3}$ & 10.2107 & -0.001 \\
Leung and Guo [27] $T_{2}$ & 10.213682 & 0.0286 \\
Feng and Chen [23] $T_{2}$ & 10.23452 & 0.232 \\
Feng and Chen [23] $T_{3}$ & 10.1935 & -0.169 \\
Feng and Chen [23] $T_{4}$ & 10.2029 & -0.077 \\
Hu [42] $T_{2}$ & 10.247682 & 0.362 \\
Hu [42] $T_{3}$ & 10.226464 & 0.154 \\
Hu et al. [43] $T_{2}$ & 10.244058 & 0.326 \\
Ramos [44] $T_{2}$ & 10.80674 & 5.84 \\
Ramos [44] $T_{3}$ & 10.55034 & 3.33 \\
Ramos [44] $T_{2}^{\prime}$ & 10.24328 & 0.318 \\
Ramos [44] $T_{2}^{\prime \prime}$ & 10.26079 & 0.49 \\
Ma et al. [3] $T_{2}$ & 10.146603 & -2.27 \\
\hline
\end{tabular}

$$
\begin{aligned}
& \cdot \sin (1.2307019873 \cdot t) \\
& -0.0119562711384 \\
& \cdot \sin (1.84605298095 \cdot t) \\
& -\left(9.51418832279 \cdot 10^{-11}\right) \\
& \cdot \sin (2.4614039746 \cdot t) \\
& +\left(8.93050847544 \cdot 10^{-05}\right) \\
& \cdot \sin (3.07675496825 \cdot t) .
\end{aligned}
$$

The approximate frequency and period are $\omega_{5}^{0}=$ $0.61535099365, T_{5}^{0}=10.2107339908715$, with an error of -0.000264516312410058 .

Table 6 presents the comparison of the absolute errors (computed as the difference in absolute value between the approximate solution and the corresponding numerical solution given by the Runge-Kutta method) corresponding to the approximate solutions $x_{\mathrm{RHB}}$ from [27] and our approximate solutions $\tilde{x}_{5}$ for the case $B=0.5$. In this case we omitted the graphical representation of the approximate solutions $x_{\mathrm{RHB}}$ and $\tilde{x}_{5}$ since they are both very close to the numerical solution.

It is easy to see from the Tables 5 and 6 that the solutions obtained by using FLSM are more accurate than the ones computed by using other methods. This fact is emphasized by Table 7 which presents a comparison of approximate periods computed in several papers, as presented in [27]. In this table, $T_{k}$ denotes an approximate period obtained by means of an approximate solution containing terms up to $\sin (k \cdot \omega \cdot t)$.

\section{Conclusions}

In the present paper the Fourier-least squares method (FLSM) is introduced as a straightforward and efficient method to compute approximate periodic solutions for a very general class of nonlinear differential equations modeling oscillatory phenomena. Since (1) is a very general one, being able to model a large class of oscillatory phenomena, FLSM can be considered a powerful and useful method.

The test problems include the cubic Duffing oscillator, the Van der Pol oscillator, and the Jerk equation. The computation of approximate solutions by FLSM clearly illustrates the accuracy of the method by comparison with approximate solutions previously computed by using other methods.

\section{Conflict of Interests}

The authors declare that there is no conflict of interests regarding the publication of this paper.

\section{References}

[1] J.-H. He, "The homotopy perturbation method nonlinear oscillators with discontinuities," Applied Mathematics and Computation, vol. 151, no. 1, pp. 287-292, 2004.

[2] M. H. Pashai, I. Khatami, and N. Tolou, "Comparative vibration analysis of a parametrically nonlinear excited oscillator using HPM and numerical method," Mathematical Problems in Engineering, vol. 2008, Article ID 956170, 11 pages, 2008.

[3] X. Ma, L. Wei, and Z. Guo, "He's homotopy perturbation method to periodic solutions of nonlinear Jerk equations," Journal of Sound and Vibration, vol. 314, no. 1-2, pp. 217-227, 2008.

[4] S. S. Ganji, D. D. Ganji, M. G. Sfahani, and S. Karimpour, "Application of AFF and HPM to the systems of strongly 
nonlinear oscillation," Current Applied Physics, vol. 10, no. 5, pp. 1317-1325, 2010.

[5] Y. Khan, M. Akbarzade, and A. Kargar, "Coupling of homotopy and the variational approach for a conservative oscillator with strong odd-nonlinearity," Scientia Iranica A, vol. 19, no. 3, pp. 417-422, 2012.

[6] J.-H. He, "Variational approach for nonlinear oscillators," Chaos, Solitons \& Fractals, vol. 34, no. 5, pp. 1430-1439, 2007.

[7] M. O. Kaya, S. Altay Demirbağ, and F. Özen Zengin, "Higherorder approximate periodic solutions of a nonlinear oscillator with discontinuity by variational approach," Mathematical Problems in Engineering, vol. 2009, Article ID 450862, 9 pages, 2009.

[8] H. P. W. Gottlieb, "Harmonic balance approach to periodic solutions of non-linear jerk equations," Journal of Sound and Vibration, vol. 271, no. 3-5, pp. 671-683, 2004.

[9] H. P. W. Gottlieb, "Harmonic balance approach for a degenerate torus of a nonlinear jerk equation," Journal of Sound and Vibration, vol. 322, no. 4-5, pp. 1005-1008, 2009.

[10] S. Telli and O. Kopmaz, "Free vibrations of a mass grounded by linear and nonlinear springs in series," Journal of Sound and Vibration, vol. 289, no. 4-5, pp. 689-710, 2006.

[11] A. Beléndez, A. Hernández, T. Beléndez et al., "Application of the harmonic balance method to a nonlinear oscillator typified by a mass attached to a stretched wire," Journal of Sound and Vibration, vol. 302, no. 4-5, pp. 1018-1029, 2007.

[12] A. Beléndez, E. Gimeno, T. Beléndez, and A. Hernández, "Rational harmonic balance based method for conservative nonlinear oscillators: application to the Duffing equation," Mechanics Research Communications, vol. 36, no. 6, pp. 728734, 2009.

[13] S. K. Lai, C. W. Lim, B. S. Wu, C. Wang, Q. C. Zeng, and X. F. He, "Newton-harmonic balancing approach for accurate solutions to nonlinear cubic-quintic Duffing oscillators," Applied Mathematical Modelling, vol. 33, no. 2, pp. 852-866, 2009.

[14] B. Ahmad and B. S. Alghamdi, "Approximation of solutions of the nonlinear Duffing equation involving both integral and non-integral forcing terms with separated boundary conditions," Computer Physics Communications, vol. 179, no. 6, pp. 409-416, 2008.

[15] H. Yao, "Solution of the Duffing equation involving both integral and non-integral forcing terms," Computer Physics Communications, vol. 180, no. 9, pp. 1481-1488, 2009.

[16] S. Ghosh, A. Roy, and D. Roy, "An adaptation of Adomian decomposition for numeric-analytic integration of strongly nonlinear and chaotic oscillators," Computer Methods in Applied Mechanics and Engineering, vol. 196, no. 4-6, pp. 1133-1153, 2007.

[17] L. Xu, "Application of He's parameter-expansion method to an oscillation of a mass attached to a stretched elastic wire," Physics Letters, Section A: General, Atomic and Solid State Physics, vol. 368, no. 3-4, pp. 259-262, 2007.

[18] S. Durmaz, S. Altay Demirbağ, and M. O. Kaya, "Approximate solutions for nonlinear oscillation of a mass attached to a stretched elastic wire," Computers \& Mathematics with Applications, vol. 61, no. 3, pp. 578-585, 2011.

[19] V. Marinca and N. Herişanu, "Periodic solutions of Duffing equation with strong non-linearity," Chaos, Solitons and Fractals, vol. 37, no. 1, pp. 144-149, 2008.

[20] N. Jamshidi and D. D. Ganji, "Application of energy balance method and variational iteration method to an oscillation of a mass attached to a stretched elastic wire," Current Applied Physics, vol. 10, no. 2, pp. 484-486, 2010.
[21] F. Geng, "A piecewise variational iteration method for treating a nonlinear oscillator of a mass attached to a stretched elastic wire," Computers \& Mathematics with Applications, vol. 62, no. 4, pp. 1641-1644, 2011.

[22] D. D. Ganji, M. Gorji, S. Soleimani, and M. Esmaeilpour, "Solution of nonlinear cubic-quintic Duffing oscillators using He's Energy Balance Method," Journal of Zhejiang University: Science A, vol. 10, no. 9, pp. 1263-1268, 2009.

[23] S.-D. Feng and L.-Q. Chen, "Homotopy analysis approach to periodic solutions of a nonlinear jerk equation," Chinese Physics Letters, vol. 26, no. 12, Article ID 124501, 2009.

[24] S. J. Liao, "Homotopy analysis method: a new analytic method for nonlinear problems," Applied Mathematics and Mechanics, vol. 19, no. 10, pp. 885-890, 1998.

[25] Y. H. Qian, S. K. Lai, W. Zhang, and Y. Xiang, "Study on asymptotic analytical solutions using HAM for strongly nonlinear vibrations of a restrained cantilever beam with an intermediate lumped mass," Numerical Algorithms, vol. 58, no. 3, pp. 293-314, 2011.

[26] A. Golbabai, M. Fardi, and K. Sayevand, "Application of the optimal homotopy asymptotic method for solving a strongly nonlinear oscillatory system," Mathematical and Computer Modelling, vol. 58, no. 11-12, pp. 1837-1843, 2012.

[27] A. Y. T. Leung and Z. Guo, "Residue harmonic balance approach to limit cycles of non-linear jerk equations," International Journal of Non-Linear Mechanics, vol. 46, no. 6, pp. 898-906, 2011.

[28] A. Elías-Zúñiga, O. Martínez-Romero, and R. K. Córdoba-Díaz, "Approximate solution for the Duffing-harmonic oscillator by the enhanced cubication method," Mathematical Problems in Engineering, vol. 2012, Article ID 618750, 12 pages, 2012.

[29] S. S. Motsa and P. Sibanda, "A note on the solutions of the Van der Pol and Duffing equations using a linearisation method," Mathematical Problems in Engineering, vol. 2012, Article ID 693453, 10 pages, 2012.

[30] J. Awrejcewicz, "Numerical investigations of the constant and periodic motions of the human vocal cords including stability and bifurcation phenomena," Dynamics and Stability of Systems, vol. 5, no. 1, pp. 11-28, 1990.

[31] I. Andrianov and J. Awrejcewicz, "A role of initial conditions choice on the results obtained using different perturbation methods," Journal of Sound and Vibration, vol. 236, no. 1, pp. 161-165, 2000.

[32] X. Wu and B. Wang, "Multidimensional adapted Runge-KuttaNyström methods for oscillatory systems," Computer Physics Communications. An International Journal and Program Library for Computational Physics and Physical Chemistry, vol. 181, no. 12, pp. 1955-1962, 2010.

[33] A. A. Kosti, Z. A. Anastassi, and T. E. Simos, "An optimized explicit Runge-Kutta-Nyström method for the numerical solution of orbital and related periodical initial value problems," Computer Physics Communications, vol. 183, no. 3, pp. 470-479, 2012.

[34] W. Shi and X. Wu, "On symplectic and symmetric ARKN methods," Computer Physics Communications, vol. 183, no. 6, pp. 1250-1258, 2012.

[35] Z. Chen, X. You, W. Shi, and Z. Liu, "Symmetric and symplectic ERKN methods for oscillatory Hamiltonian systems," Computer Physics Communications, vol. 183, no. 1, pp. 86-98, 2012.

[36] V. P. Chua and M. Porter, "Cubic-quintic duffing oscillator," in Proceedings of the Annual Conference on Chaos and Nonlinear Dynamics, Dynamic Days, 2004. 
[37] T. Kalmár-Nagy and B. Balachandran, "Forced harmonic vibration of a Duffing oscillator with linear viscous damping," in The Duffing Equation: Nonlinear Oscillators and their Behaviour, I. Kovacic and M. J. Brennan, Eds., pp. 139-174, John Wiley and Sons, 2011.

[38] J. Awrejcewicz, "Numerical versus analytical conditions for chaos, using the example of the Duffing oscillator," Journal of the Physical Society of Japan, vol. 60, no. 3, pp. 785-788, 1991.

[39] V. Patidar and K. K. Sud, "Bifurcation and chaos in simple jerk dynamical systems," Pramana - Journal of Physics, vol. 64, no. 1, pp. 75-93, 2005.

[40] H. P. W. Gottlieb, "Harmonic balance approach to limit cycles for nonlinear jerk equations," Journal of Sound and Vibration, vol. 297, no. 1-2, pp. 243-250, 2006.

[41] B. S. Wu, C. W. Lim, and W. P. Sun, "Improved harmonic balance approach to periodic solutions of non-linear jerk equations," Physics Letters, Section A: General, Atomic and Solid State Physics, vol. 354, no. 1-2, pp. 95-100, 2006.

[42] H. Hu, "Perturbation method for periodic solutions of nonlinear jerk equations," Physics Letters A, vol. 372, no. 23, pp. 42054209, 2008.

[43] H. Hu, M. Y. Zheng, and Y. J. Guo, "Iteration calculations of periodic solutions to nonlinear jerk equations," Acta Mechanica, vol. 209, no. 3-4, pp. 269-274, 2010.

[44] J. I. Ramos, "Analytical and approximate solutions to autonomous, nonlinear, third-order ordinary differential equations," Nonlinear Analysis: Real World Applications, vol. 11, no. 3, pp. 1613-1626, 2010. 


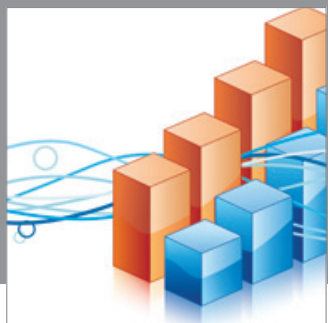

Advances in

Operations Research

mansans

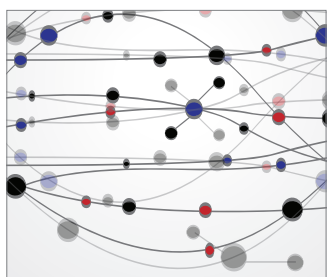

The Scientific World Journal
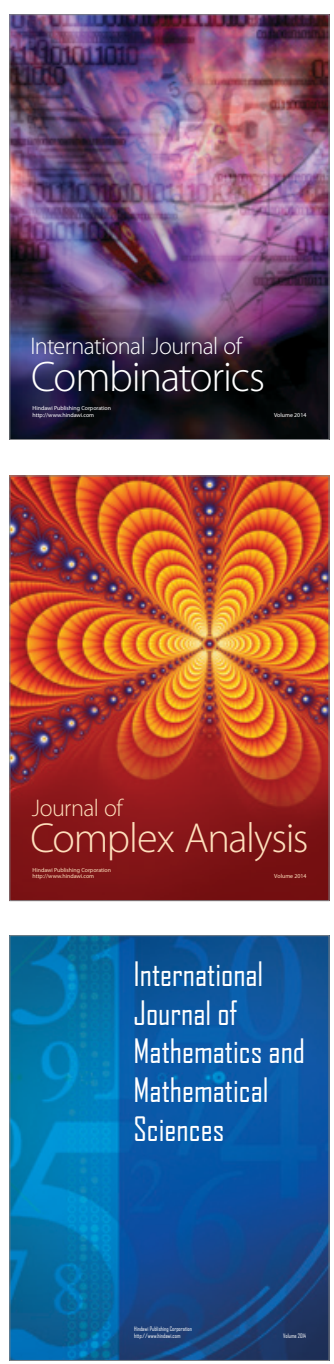
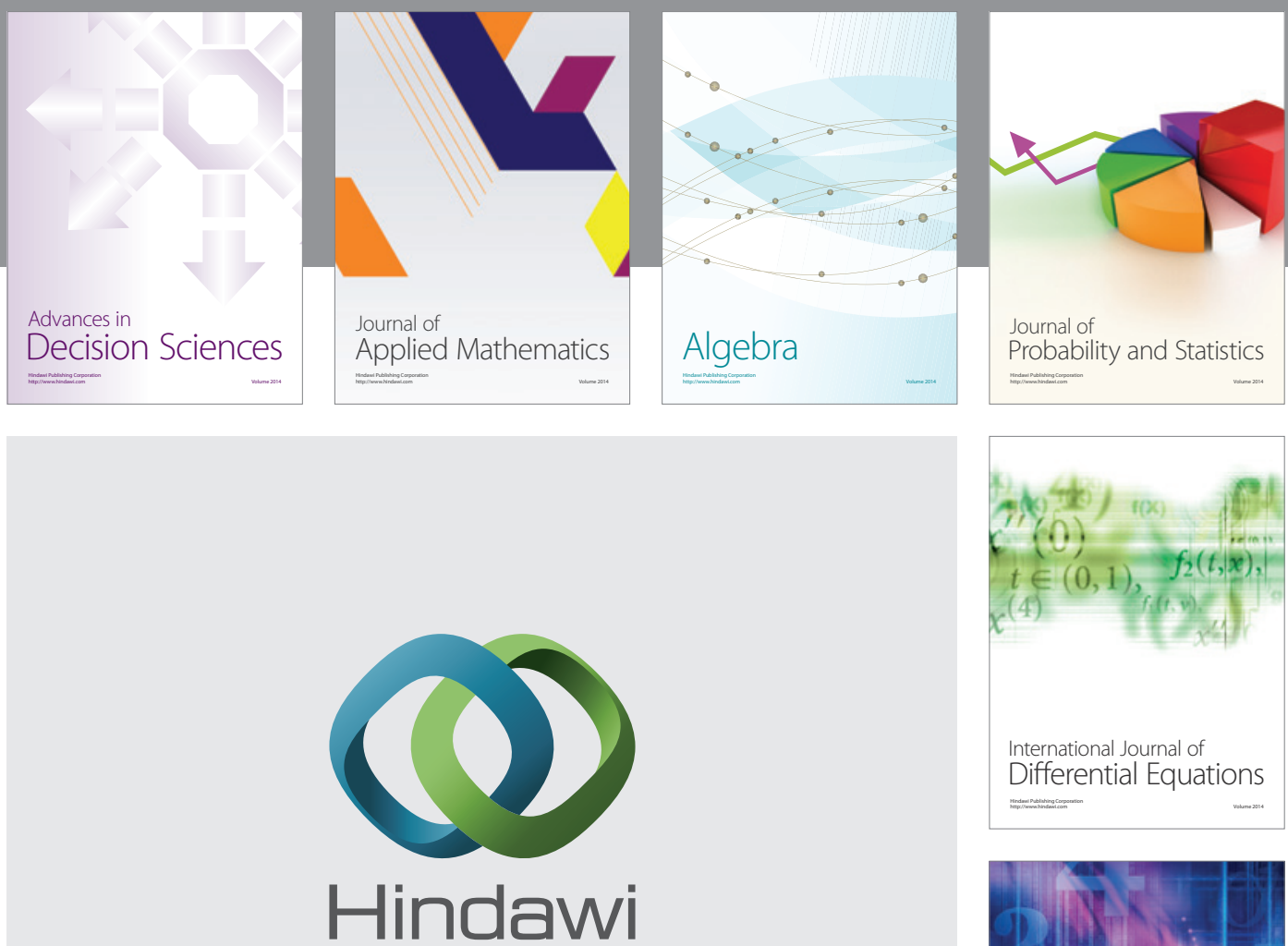

Submit your manuscripts at http://www.hindawi.com
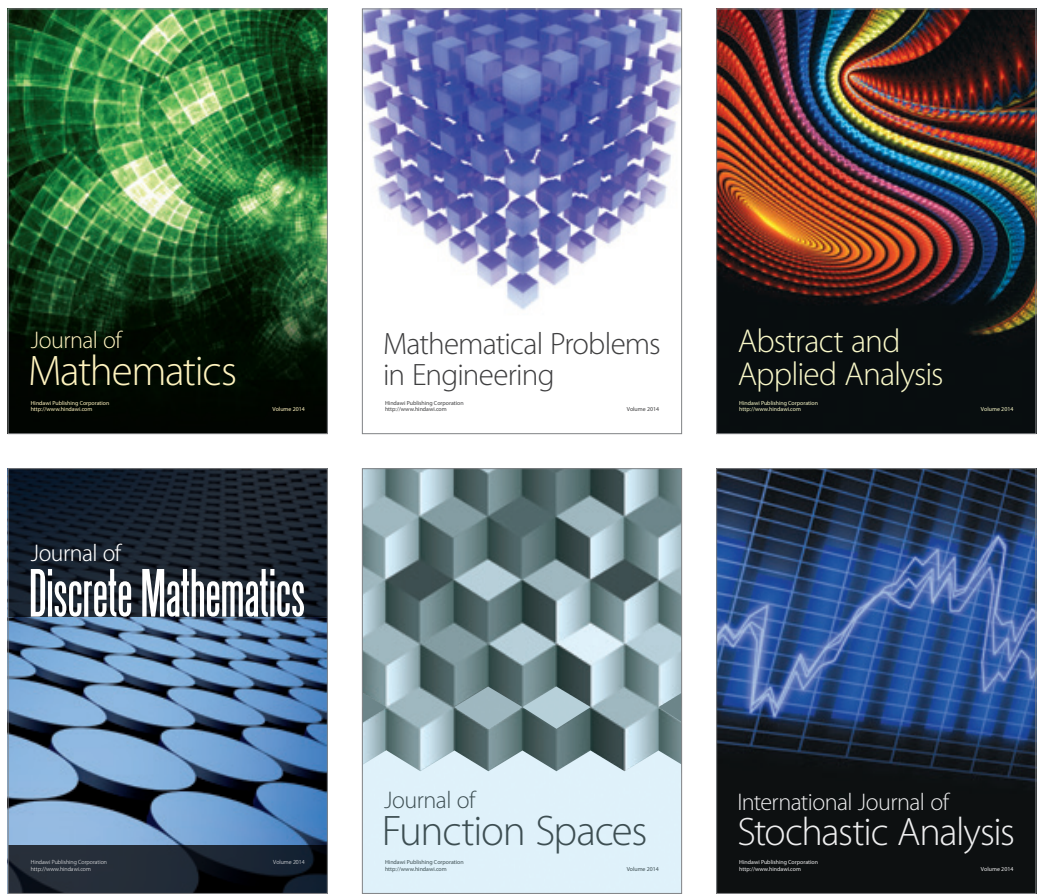

Journal of

Function Spaces

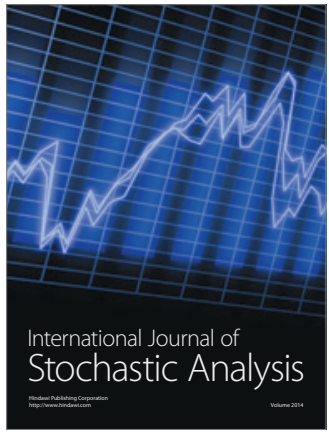

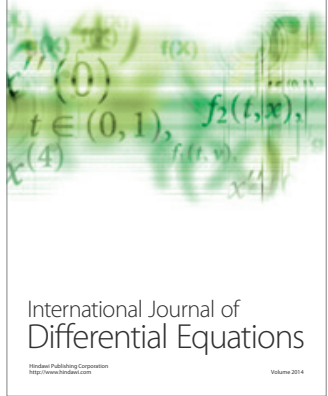
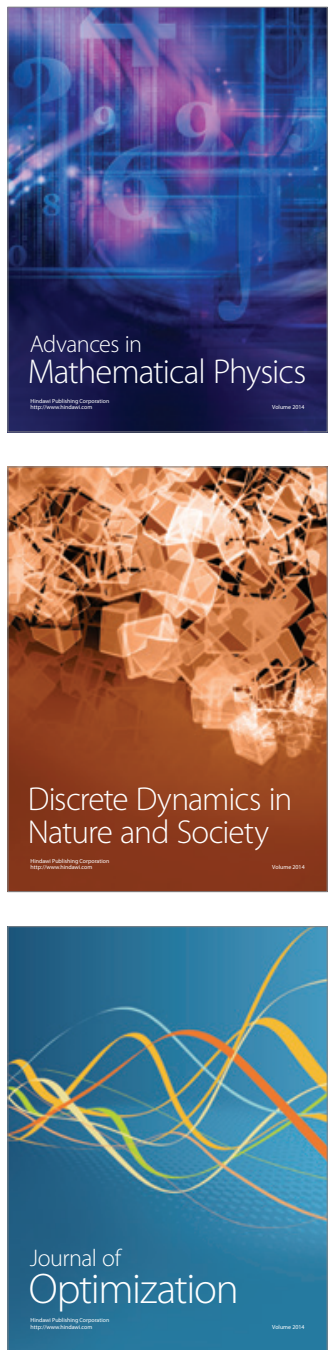